\title{
Osteopathie für Kleintiere
}

\section{Der sanfte Weg zu mehr Beweglichkeit und Ausgeglichenheit}

\section{Sophia Strauß}

Die Osteopathie wirkt oft sehr unspektakulär - keine Geräte, kein schnelles, kraftvolles Einrenken. Stattdessen sanfte, minimale Handbewegungen, die eine tiefe Entspannung im Tier bewirken. Wir zeigen, was hinter dieser „unscheinbaren“ und doch sehr tiefgreifenden Behandlung steckt!

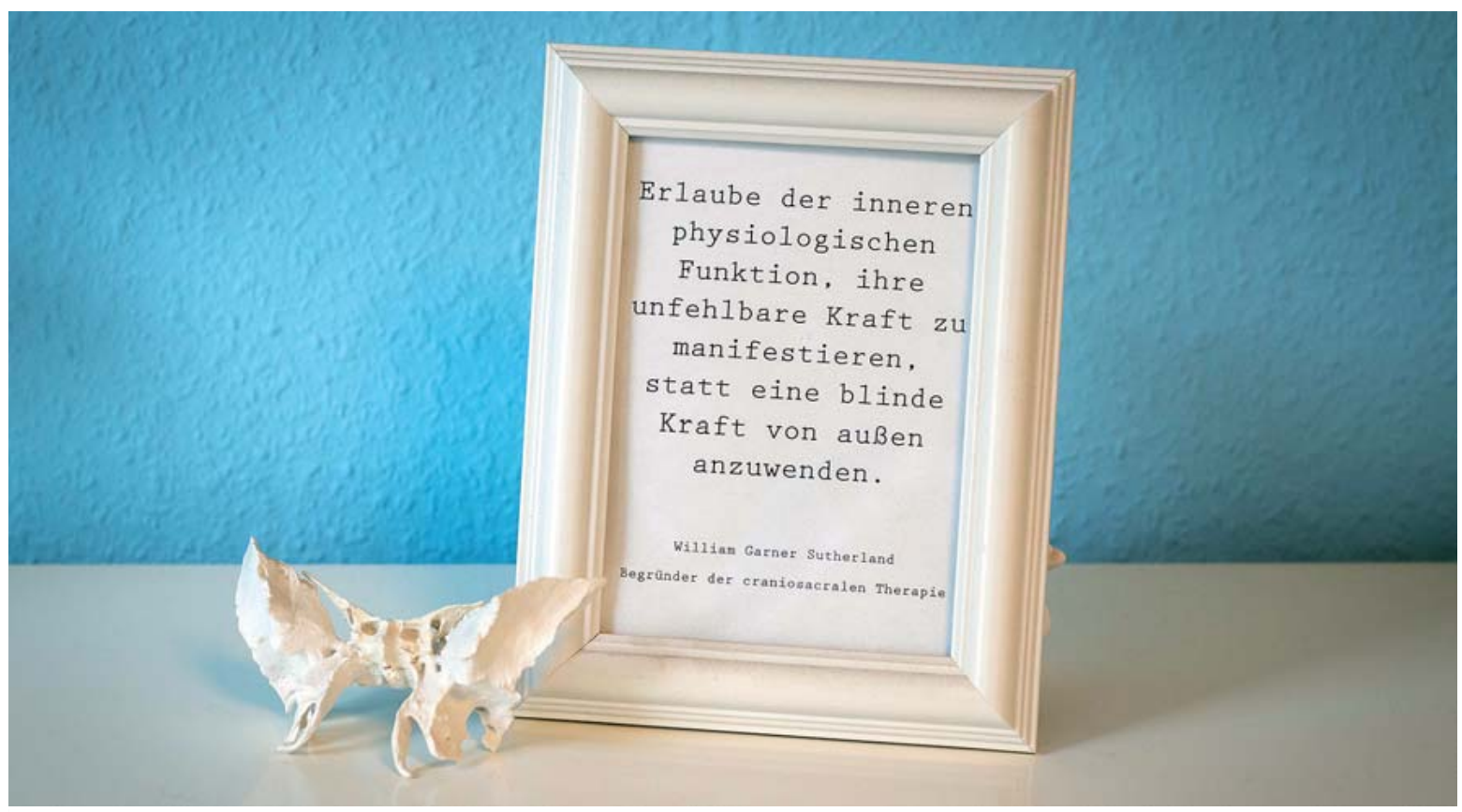

Quelle: S. Strauß

\section{Grundlagen der Osteopathie}

Die Osteopathie ist eine ganzheitliche, manuelle Therapieform zur Untersuchung und Behandlung von Funktionsstörungen, die vor circa 140 Jahren in den USA von dem Amerikaner Dr. Andrew Taylor Still begründet wurde. Er erkannte, dass Krankheiten mit einer verminderten Eigenbewegung in Strukturen wie Muskeln, Faszien, Bändern, Gelenken, Knochen und Organen einhergeht. Das Gedankengut der Osteopathie beruht im Wesentlichen auf dem Ansatz, dass jedes Individuum eine Einheit aus Körper, Geist und Seele darstellt, die in der Lage ist, sich selbst zu regulieren. In dieser Einheit beeinflussen sich Struktur und Funktion wechselseitig.
Unabhängig von bestehenden Diagnosen untersucht der Osteopath alle Bereiche des Körpers auf Spannungen und Bewegungseinschränkungen und deren Verkettungen untereinander, mit dem Ziel, die Ursache zu finden, welche die Regulationsprozesse im Organismus einschränkt. Dabei unterscheidet man drei Teilbereiche: parietale, viszerale und kraniosakrale Osteopathie.

\section{Die drei Teilbereiche}

Alle drei Systeme des Körpers stehen in unmittelbarer Beziehung zueinander und dürfen nicht isoliert voneinander betrachtet werden. Insbesondere bei chronischen Erkrankungen befinden sich die Spannungszustände durch Verkettungen oftmals in mehreren Strukturen und Körpersystemen. Erst das harmonische Zusammenspiel aller ge- 
nannten Systeme ermöglicht dem Körper, als Einheit zu funktionieren.

Die parietale Osteopathie beschäftigt sich mit dem Bewegungsapparat: den Knochen, Gelenken und Muskeln. Ein spezieller Fokus liegt dabei auf den Faszien - dem Trenngewebe bzw. Stützgerüst des Körpers -, welches unter anderem die Bewegung der einzelnen Strukturen gegeneinander ermöglicht.

Die viszerale Osteopathie erfasst und behandelt Spannungsänderungen in den Bauch-, Becken- und Thoraxorganen ( $\triangleright$ Abb. 1). Im Fokus stehen dabei die Eigenbewegung, das Befestigungssystem inklusive der Bänder und die Beweglichkeit der Organe zueinander. Die viszerale Osteopathie wurde erst vor ca. 40 Jahren v. a. durch die Arbeit des französischen Osteopathen Jean-Pierre Barral in Europa bekannt.

Die kraniosakrale Therapie geht auf William Garner Sutherland zurück und beschäftigt sich mit der freien Beweglichkeit aller Schädelknochen, der Wirbelsäule, dem Sakrum sowie aller dazugehörigen Membranen ( $\mathbf{A}$ bb. 2). Der Schädel und das Kreuzbein werden dabei als Pole betrachtet, die über ein Membransystem miteinander verbunden sind und sich synchron zueinander bewegen.

\section{Spannungen lösen}

Das Ziel der Osteopathie ist das Lösen von Spannungszuständen im Gewebe und das Gleichgewicht des gesamten Organismus auf allen Ebenen ( $>$ Abb. 3). Jeder Körper ist stets gewillt, gesund zu sein. Hilft man, dass alle Strukturen ihre normale Bewegung und Funktion durchführen können, kann eine Gesundung aufgrund der Selbstregulation eigenständig bewerkstelligt werden. Oft beginnt eine akute Lahmheit durch Spannungsänderungen im Gewebe, weit bevor sie äußerlich erkennbar ist. Was anfänglich kompensiert werden kann, wird durch bestimmte innere oder äußere Einflüsse plötzlich klinisch auffällig. Die dann wahrnehmbaren äußerlichen Krankheitssymptome sind lediglich die Grenzen der Kompensationsfähigkeit des Körpers. Wenn der Körper also nicht mehr in der Lage ist, beispielsweise ein Problem des Bewegungsapparates durch Umbelastung zu kompensieren, werden Symptome wie Lahmen sichtbar - oft jedoch dort, wo die Überlastung stattgefunden hat und nicht an der Stelle der ursächlichen Spannung.

\section{Suchen und Finden}

Die primäre Spannung und somit den Behandlungsansatz für die Therapie zu finden, ist das Ziel des osteopathischen Untersuchungsgangs. Durch gezielte Techniken werden Befunde erhoben und deren Verkettungen untereinander hierarchisch ermittelt, sodass im Idealfall nur die Primärläsion, die ursächlich den Organismus aus seinem Gleichgewicht gebracht hat, behandelt wird.

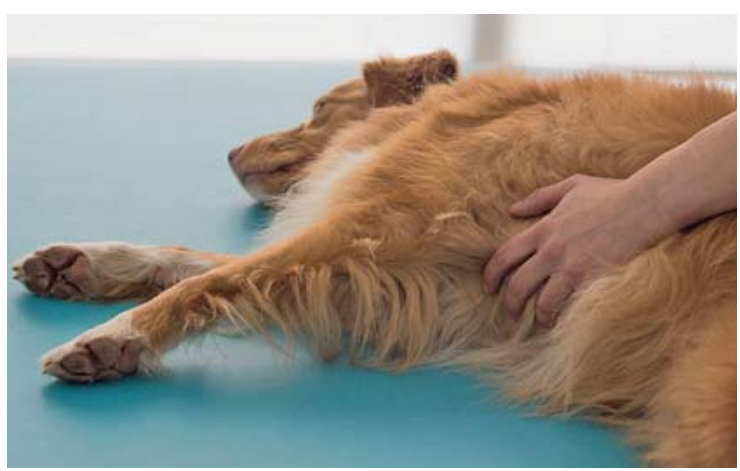

- Abb. 1 Behandlung des Zwerchfells - ein wichtiger Körperteil bei der viszeralen und auch parietalen Osteopathie. Quelle: S. Strauß

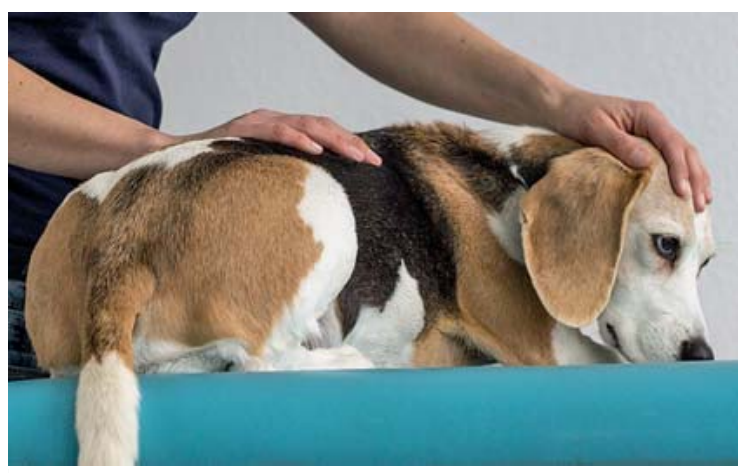

- Abb. 2 Beagle bei der Behandlung des Schädels. Durch diese Handanlage ist der kraniosakrale Rhythmus besonders gut wahrnehmbar. Quelle: S. Strauß

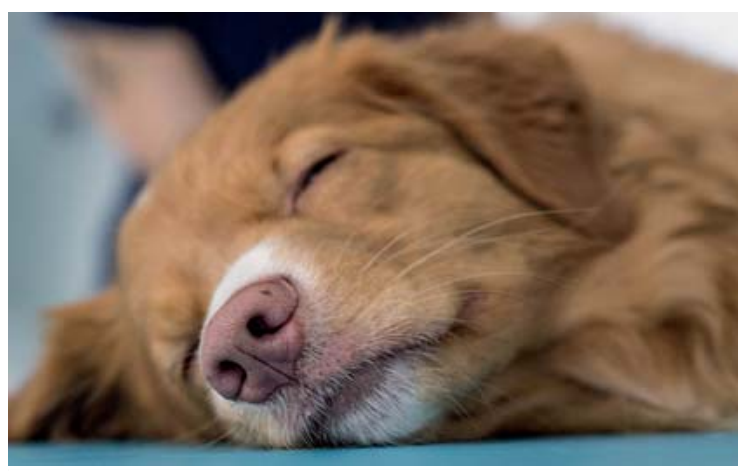

- Abb. 3 Genüssliche Entspannung bei der Behandlung: Spannung erkennen und Spannung lösen sind zwei wichtige Ziele der Osteopathie. Quelle: S. Strauß

Die Beweglichkeit aller eingeschränkten Strukturen soll wiederhergestellt, der ungehinderte Fluss der Körperflüssigkeiten gewährleistet sowie ein ausbalanciertes, stabiles Nervensystem etabliert werden. Die besondere Anforderung besteht dabei darin, jeden Patienten mit seinem gesundheitlichen Werdegang individuell zu betrachten 
und den für diesen Patienten, für diese Vorgeschichte und zu diesem Zeitpunkt bestmögliche Hilfestellung zu finden.

\section{Wo hilft Osteopathie?}

Unabhängig von Indikationen gemäß schulmedizinischer Diagnosen kann Osteopathie als ganzheitliche Therapie jedem Lebewesen helfen, allägliche Belastungen leichter zu bewältigen. Durch die Mobilisierung der Selbstheilungskräfte bzw. durch die Anregung der körpereigenen Regulationsmechanismen in allen drei Systemen des Körpers (parietales, viszerales und kraniosakrales System) übt die Osteopathie eine positive, unterstützende Wirkung auf nahezu jede Erkrankung aus.

\section{Indikationen}

Klassische Indikationen für eine osteopathische Behandlung sind Erkrankungen des Bewegungsapparates wie beispielsweise Arthrose, Spondylose, Kreuzbandriss, HD oder ED, aber auch neurologische Erkrankungen wie ein Bandscheibenvorfall oder das Cauda-Equina-Kompressionssyndrom. Oft sind es jedoch unklare Lahmheiten und schulmedizinisch austherapierte Hunde, die ihren Weg in die Osteopathie-Praxis finden. Weitere Indikationen sind Symptomatiken im Bereich des Schädels, wie zum Beispiel Kiefergelenksprobleme und auch postoperative und posttraumatische Behandlungen. Auch bei Störungen im Organsystem wie beispielsweise chronischen MagenDarm-Problemen oder auch Inkontinenz kann eine osteopathische Behandlung eine sinnvolle Unterstützung sein. Und nicht zuletzt können auch seelische Aspekte wie psychische Traumata oder Verhaltensauffälligkeiten eine Indikation darstellen.

\section{Merke}

Bei schweren akuten und fieberhaften Erkrankungen darf Osteopathie nicht stattfinden!

\section{Einschränkungen}

Akute Erkrankungen, wie zum Beispiel Infektionen, Fieber, Krebserkrankungen oder schwere Herzerkrankungen, gehören nicht in den Behandlungsbereich der Osteopathie.

Gibt es bereits darstellbare strukturelle Veränderungen (z. B. Arthrose), so sind dem optimalen Therapieziel, einer dauerhaften Beschwerdefreiheit, Grenzen gesetzt. Jedoch profitieren insbesondere die chronisch erkrankten Vierbeiner von der Osteopathie, da in diesen Fällen der Körper immer wieder aufgrund der bestehenden Befunde in seinen Kompensationsmöglichkeiten gefordert wird. Die osteopathischen Behandlungen geben Kapazitäten zur Kompensation wieder frei, indem überlastete Strukturen entlastet und weitere, durch Umbelastung entstandene Verkettungen reduziert werden. So kann bei chronisch erkrankten Patienten das klinische Bild erheblich verbessert und idealerweise auch auf die Gabe von Schmerzmedikamenten verzichtet werden.

\section{Osteopathie praktisch}

Zu Beginn einer jeden osteopathischen Therapie erfolgt eine ausführliche Befunderhebung, die sich deutlich von der schulmedizinischen Diagnose unterscheidet: Osteopathische Befunde werden durch einen rein manuellen Untersuchungsgang erhoben, indem der gesamte Organismus auf Bewegungseinschränkungen, Asymmetrien und Gewebespannungen in allen drei Körpersystemen untersucht wird. Zusätzlich werden eventuelle Verkettungen der ermittelten Befunde untereinander geprüft, um die Primärläsion und somit den Hauptansatzpunkt für die Therapie zu finden.

Gleichzeitig ist neben der manuellen Untersuchung auch die Krankheitsgeschichte des Patienten von Interesse: Aktuelle Symptome sind dabei genauso wichtig wie vorangegangene Operationen, Erkrankungen, Unfälle oder Traumata. Sie alle hinterlassen Spuren im Körper und tragen möglicherweise zur aktuellen Symptomatik bei oder stellen sogar die primäre Ursache dar.

\section{Individuelle Behandlung}

Die anschließende Behandlung konzentriert sich in erster Linie auf den ermittelten Befund mit der höchsten Priorität. Hierfür stehen dem Therapeuten verschiedene Behandlungstechniken zur Verfügung, welche die körpereigenen Regulationsmechanismen ansprechen und mit deren Hilfe die Lösung der Dysfunktionen erreicht werden kann.

Oft ist der osteopathische Behandlungsansatz an einer ganz anderen Stelle zu finden als dort, wo das Problem sichtbar geworden ist, und jeder Fall ist hierbei individuell zu betrachten. Patienten mit der gleichen Symptomatik können aufgrund ihrer eigenen Vorgeschichte, ihrer Gesamtkonstitution und ihrer individuellen Möglichkeiten der Kompensation eine sehr unterschiedliche Behandlung benötigen.

AUSBILDUNG IM BEREICH OSTEOPATHIE Derzeit gibt es in Deutschland weder eine Vereinheitlichung von Ausbildungsinhalten noch gesetzliche Regelungen zur Berufsbezeichnung des KleintierOsteopathen. Dies erschwert Patientenbesitzern und überweisenden Tierarztpraxen oft, konkrete Therapeuten-Empfehlungen auszusprechen. Und es gibt auch Missverständnisse oder Unklarheiten, was Osteopathie genau ist und für wen sie geeignet ist. Tipps und Adressen zum Thema sind bei der Autorin erhältlich!

\section{Unser Praxisfall}

Eine 6-jährige Australian Shepherd Hündin erlitt aus der Bewegung heraus eine plötzliche Lähmung der Hinter- 
gliedmaßen, doch die genaue Ursache blieb ungeklärt. Durch die tierärztliche Therapie war die Hündin innerhalb kurzer Zeit wieder normal gehfähig. Sie zeigte jedoch auch sechs Wochen nach dem Akutgeschehen Probleme beim Aufstehen, diagonales Laufen im Trab und wirkte laut Besitzerin insgesamt schwerfällig. Das Treppenlaufen sowie Sprünge ins Auto waren sehr mühsam. In der Vergangenheit hatte die Hündin zweimal eine Pyometra, weswegen sie kastriert wurde.

\section{Erstuntersuchung und Behandlung}

Im Untersuchungsgang zeigt sich, dass sowohl Brust- als auch Lendenwirbelsäule in der dorsoventralen Federung sehr viel Spannung aufweisen. Die Thoraxrotation ist eingeschränkt, das Becken ist unbeweglich. Die Muskulatur der Brustwirbelsäule hat deutliche Verspannungen. Insgesamt ist der Körperschwerpunkt stark nach vorn verlagert. Die Hintergliedmaßen werden deutlich entlastet.

In der genaueren osteopathischen Untersuchung lässt sich eine deutliche Einschränkung des kraniosakralen Rhythmus im Bereich des Sakrums feststellen, welche sich durch genauere Untersuchung mit Spannungen im Bereich der Lendenwirbelsäule, insbesondere L2, L3 sowie L7 in Verbindung bringen lässt. Die muskulären Spannungen in der Brustwirbelsäule lassen sich durch gezielte Techniken auf eine Problematik im Bereich der Beckenorgane zurückführen.

Es wird ein myofasziales Release im Bereich der kaudalen Lendenwirbelsäule durchgeführt sowie eine kraniosakrale Behandlung des Sakrums. Da die Hündin anfänglich sehr gestresst und nervös wirkt, wird die Behandlung zur positiven Verknüpfung vorzeitig beendet, nachdem sich die Hündin durch die genannten Behandlungen sichtbar entspannt hat.

\section{Nach 14, 28 und 60 Tagen}

Die Hündin läuft bereits lockerer, Aufstehen und Treppenlaufen sind noch unverändert. Die genauere Untersuchung der kranialen Lendenwirbelsäule zeigt eine Beteiligung der linken Niere an, die in ihrer Motilität eingeschränkt ist und entsprechend behandelt wird. Zusätzlich wird im viszeralen Bereich die Kastrationsnarbe sowie im kraniosakralen System das Sakrum und der atlanto-okzipitale Übergang behandelt.

Nach 28 Tagen steht die Hündin ohne Beschwerden auf, Treppen läuft sie noch langsam, und das Gewicht wird immer noch leicht auf die Vordergliedmaßen verlagert. Die dorsoventrale Federung der Lendenwirbelsäule hat sich erheblich verbessert, und das Becken ist locker. Es zeigt sich nun eine Spannung in einem zentralen Bereich des Schädels, und die Hündin wird an der SSO (Synchondrosis sphenooccipitalis) sowie im Übergangsbereich zwischen dem 7. Lendenwirbel und dem Sakrum behandelt.
Zwei Monate nach Therapiebeginn zeigt die Hündin im Alltag keine Probleme mehr: Die Gewichtsverteilung ist gleichmäßig, die Wirbelsäule ist locker und sowohl Becken als auch Thorax sind gut beweglich. Die Besitzerin möchte den Hund nun prophylaktisch in längeren Abständen vorstellen, und es bestätigt sich in weiteren Folgeterminen, dass die Anfangsbefunde dauerhaft gelöst werden konnten.

\section{Analyse}

In dem dargestellten Fall zeigt sich eine Verkettung innerhalb des kraniosakralen Systems sowie eine Verkettung zwischen parietalem und viszeralem System. Durch eine Spannungsweiterleitung über die Rückenmarkshäute wurde ausgehend von der SSO Zug auf das Sakrum ausgeübt. Die Lendenwirbelsäule war sowohl durch das Sakrum als auch unter Beteiligung der linken Niere eingeschränkt. Das Becken konnte aufgrund der Kastrationsnarbe nicht von allein in eine freie Beweglichkeit zurückfinden. Einige Befunde können womöglich durch das Akutgeschehen verursacht worden sein - andere, insbesondere die Kastrationsnarbe, konnten bis zu diesem Zeitpunkt offenbar gut kompensiert werden.

Der geschilderte Fall zeigt eine relativ schnelle Genesung, wobei der zeitnahe Therapiebeginn nach der Akutphase sicher dazu beiträgt. Wenn Patienten erst Monate oder teilweise Jahre nach dem Auftreten der ersten Symptome in der Osteopathie vorgestellt werden, dann bedürfen die entstandenen komplexen Spannungsmuster häufig mehrerer Behandlungseinheiten.

Merke

Osteopathie hilft bei aktuellen Problemen, bei chronischen Leiden und aktiviert Selbstheilungskräfte!

\section{Fazit}

Egal, ob akut auffällig gewordene oder chronisch erkrankte Tiere, ob Welpe oder Hundesenior - Osteopathie ist bei vielen Beschwerdebildern ein sehr erfolgsversprechender Behandlungsansatz und schenkt den Patienten nicht nur eine deutlich bessere Beweglichkeit, sondern auch mehr Lebensfreude und ein ausgeglicheneres Gemüt.

\section{Korrespondenzadresse}

\author{
Sophia Strauß \\ Hundeosteopathie Kiel \\ kontakt@hundeosteopathie-kiel.de \\ www.hundeosteopathie-kiel.de/
}

Literatur bei der Autorin erhältlich.

Bibliografie

DOI https://doi.org/10.1055/a-1012-3464

Team konkret 2019; 15: 2-5

(c) Georg Thieme Verlag KG Stuttgart · New York

ISSN 1869-3202 